\title{
Pollution Sensor Based Data Communication via Android Device
}

\author{
Kamna Ashu \\ Chandigarh Engineering College, Mohali, India
}

\begin{abstract}
This paper describes an investigation to build an Android application that gathers the air pollution information and displays information via a GPRS network. The air pollution data is collected by hardware modules transmitted to Zigbee enabled android device which further transmits to a back-end server to display the pollution map of the place. The application also displays the position of the user. Pollution is the presence in or introduction into the environment of a substance which has harmful or poisonous effects (Oxford University) [1] which certainly requires a careful study before developing any suitable Pollution mapping application. The device will regularly update pollution information and is available anytime. Nevertheless the latest attempts at introducing green technologies for all kinds of machines are starting to be successful thanks to the continuous standardization process that is pondering over the environment prices and making devices more useful for the end user. Even so several important issues are always to be handled strictly before developing and installing an Android Data communication application; factors like USB host, USB accessory, usefulness, robustness and price are critical to determine if the final product will accomplish.
\end{abstract}

Keywords: Android, USB Accessory, GSM, UART, Mapping, GPRS, USB Host

\section{Introduction}

Nowadays with more than $50 \%$ of the human population living on cities, the air pollution and the quality of life in the cities have decreased considerably. Taking into account the industries near the cities, the public and private transports, planes and power plants that involve burning of fossil fuels, the aim was to build an Android application that maps pollution data. Air pollution monitoring system in cities use sensor networks that monitor $\mathrm{CO}, \mathrm{SO} 2$ and $\mathrm{O} 3$ [2]. The system consists of an Android data acquisition unit and a Pollution monitoring server. The pollution mapping system has been established over web as Air Quality Index [3]. The system sent an alarm when the pollution level increased their threshold value.

The integrated unit is a Zigbee enabled android device and a sensor bracelet, the data acquisition unit that utilizes the wireless mobile public networks. The sensor bracelet can be worn on the wrist. While the person is on the move, the microcontroller generates a frame consisting of the acquired air pollutant level from the sensors array. The system gathered pollutant level through sensor based microcontroller and downloaded it to a remote computer for processing. The UART and USB host and Android Accessory device was initialized and the drivers were opened. After the USB enumeration 'Get Protocol' request was sent. The application then sends the identification string. The strings after being acknowledged read data from UART buffer. The received data is written to Android at the rate of one byte at a time. When the received byte was null, it was passed to message handler which converted bytes to strings.

The application sends the string received from USB Accessory (gathered from sensors) via GPRS network to a server. The device gathered the air pollution (Arduino) and the latitude, longitude and altitude (Android) every X minutes. Then the collected data was sent via HTTP POST message to the server. Along with that the GPS application sends the location of android device. If there is no Internet connection, the application would store locally the information. Once the application detects connectivity, this gathered data will be sent. The posted data was stored in the server. The data was visualized in a map with attributes such as hour, position and type of gas or gases. It is put together for 5 seconds of time and changes for more than 5 meters between updates. When the location of the user is changed, it displays the last location. The captured data was visualized through a REST API.

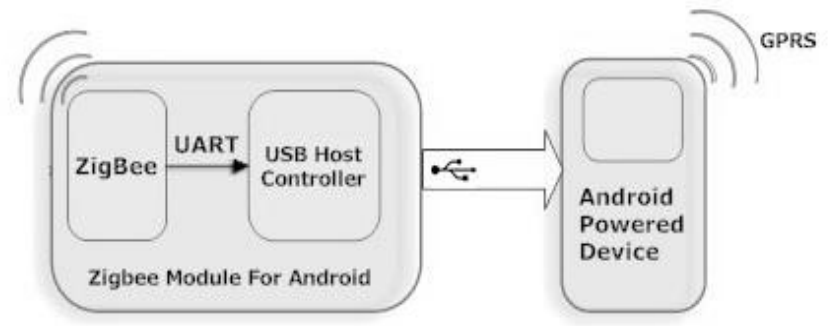

Fig 1: Block diagram of the System 


\section{System Requirements}

The functional requirements of the system are as follows.

- System needs to store the data and provide access to a location map interface.

- It should be mobile and accessible from the Internet 24/7.

- It should use minimum power.

- System must support accurate and continuous real-time data collection.

- System must support two-way communication between the client and the server.

- System must be field-configurable and should be easy to deploy.

\section{System Architecture}

The system is divided into 3 major components: (1) Sensor bracelet (2) Data Storage, and (3) Zigbee enabled android device.

A. The Sensor Bracelet: It is Zigbee enabled and is responsible for the continuous collection of the pollution data and the reliable transfer of the data to the system storage backbone. It is the Data collection unit. It consists of two main parts: (1) Pollution Data Collection and (2) Pollution Data Transfer. The component can be easily expanded to cover new areas without needing to increase the number of sensors. This can be done by simply changing the module carrier schedule to cover the new areas [3], [4]

B. The Data Storage component: It is responsible for the efficient storage and analysis of the collected pollution data and for guaranteeing access by authenticated users only.

C. The Zigbee enabled Android device: This component is responsible to allow the end user to easily access and view the pollution information via the Worldwide Web (WWW).

Data transfer is done every five seconds and changes for five metres to provide the latest pollution information to the end user. This can be achieved by either sending messages over the GSM network or GPRS internet network.

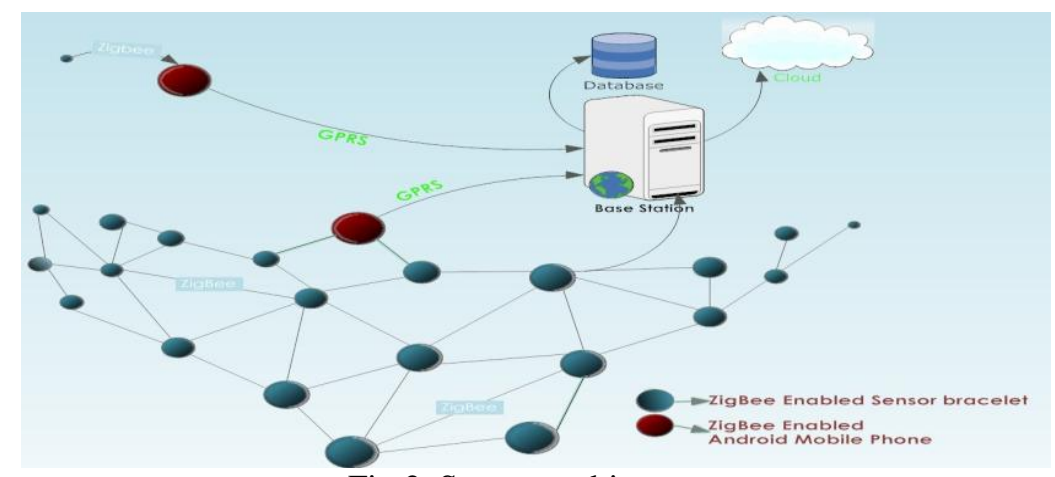

Fig 2. System architecture

The goals of the pollution maps are:

\section{Purpose}

- Map in real-time the air pollution of the city.

- Share with your friends and neighbours the state of pollution of the city in order to decide where it is recommended to go for sports or walk.

- Create social awareness of the reality of the air pollution of the city.

- To achieve these goals, pollution maps will offer an air pollution sensor which will be connected via USB to you Android device.

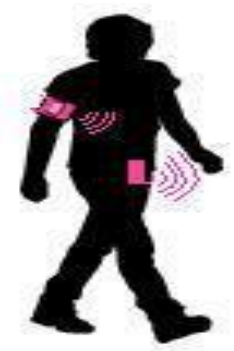

Fig3. Sensor bracelet with Android device 


\section{FLOWCHART}

FLOW CHART FOR ANDROID FOR USB COMMUNICATION \& DATA UPLOADING TO SER VER
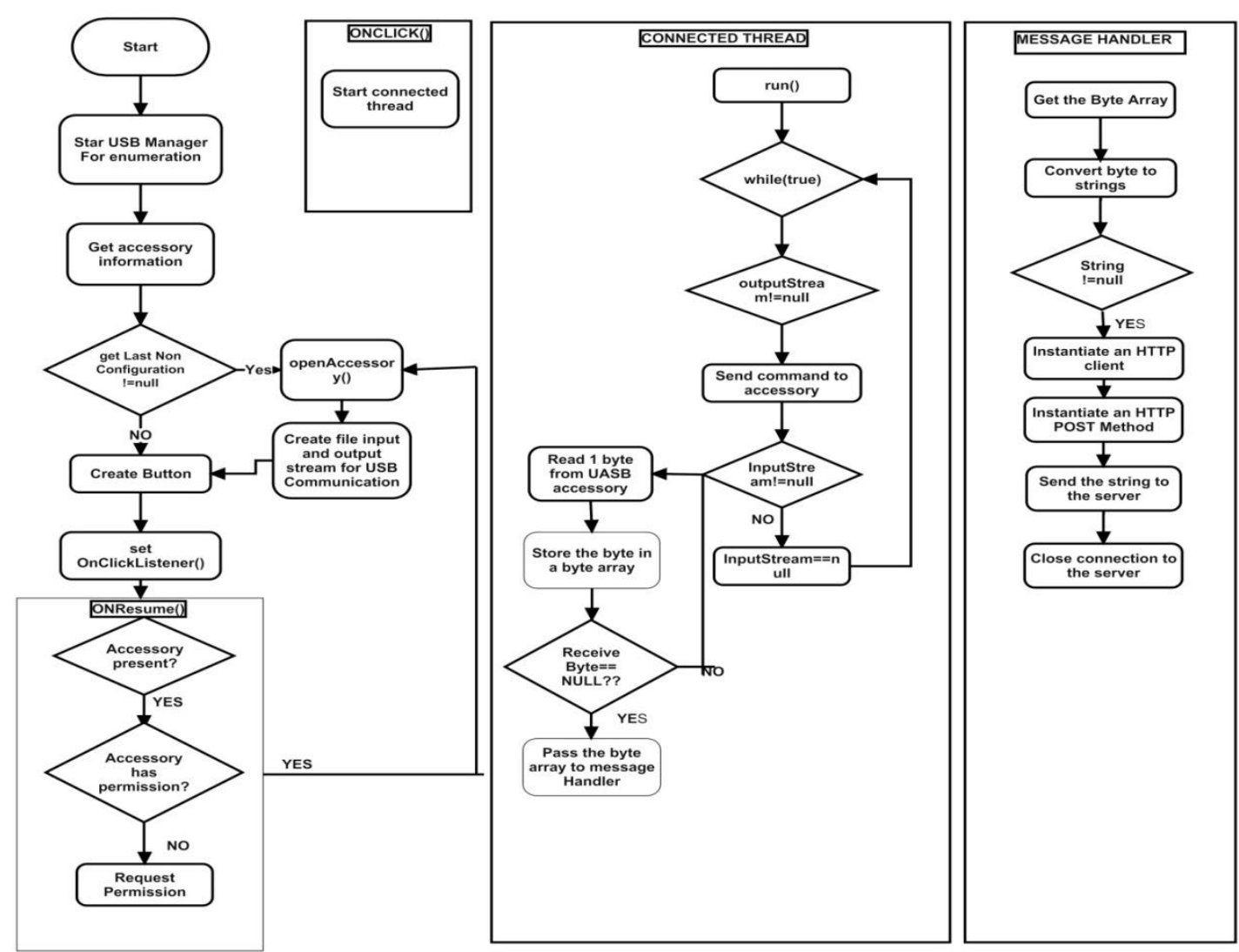

Fig 4. USB communication

\section{Conclusion}

A wireless distributed mobile air pollution monitoring system was designed, implemented and tested using the GPRS public network. The system utilizes city buses to collect pollutant gases such as $\mathrm{CO}, \mathrm{NO} 2$, and $\mathrm{SO} 2$. The pollution data from various mobile sensor arrays is transmitted to a central several that make this data available on the Internet through a Google Maps interface. The data shows the pollutant levels and their conformance to local air quality standards. It is worth mentioning that much more work is required to commercialize the system. Map in real time the air pollution of the city

\section{[1] Oxford University Press}

\section{References}

[2] N. Kularatna and B. H. Sudantha, “ environmental air pollution monitoring system based on the IEEE 1451 standard for low cos requirements," IEEE Sensors J., vol. 8, pp. 415-422, Apr. 2008.

[3 ]"The Air Quality Archive", www.airquality.co.uk

[4] Kanaroglou, P. S., Jerrett, M., Morrison, B., et. al."Establishing an air pollution monitoring network for intraurban population exposure assessment: a location-allocation approach." 